\title{
Strukturalistische vs. gebrauchsbasierte Modellierung des Erwerbs der definiten Artikel und D-Pronomen des Deutschen
}

\author{
M.E. ... [ist] ... davon auszugehen, dass analytische Fähigkeiten, die die Dekomposition \\ eines komplexen Ausdrucks ermöglichen, und Kategorisierungen, die auf holistische \\ Repräsentationen zurückzuführen sind, koexistieren. \\ (Köpcke 1993)
}

\begin{abstract}
Während in den Naturwissenschaften gegenwärtig eine starke Tendenz zur Analytik und Erfassung immer kleinerer Einheiten und ihrer Eigenschaften vorherrscht, hat sich in der Linguistik die genau gegensätzliche Orientierung weg von den Einzelbestandteilen hin zu komplexen Gebilden und deren Eigenschaften etabliert. Man kann sich zum einen fragen, wie diese gegensätzliche Entwicklung zu erklären ist und zum anderen, ob die in der aktuellen Linguistik wieder sehr präsente Haltung des Entweder-Oder, d. h. entweder strukturalistische oder gebrauchsbasierte Erklärung der sprachlichen Strukturen gerechtfertigt ist. Das als Motto für diesen Aufsatz gewählte Zitat aus der Habilitationsschrift von Klaus-Michael Köpcke zeigt, es war nicht immer und von vornherein so.

Mein Beitrag verfolgt das Ziel, die Erklärungskraft von Schema-/usage-based bzw. konstruktionsgrammatischen Ansätzen für - in anderen theoretischen Modellen als paradigmatisch verstandene - morphologische Strukturen zu prüfen. Es geht dabei letztlich um die Frage, ob gebrauchsorientierte Modelle den Anspruch einer Theorie der mentalen Organisation des sprachlichen Wissens erfüllen können. Der Gegenstand, an dem diese Diskussion exemplarisch geführt wird, ist der L1-Erwerb der definiten Artikel und Pronomen (D-Formen im Folgenden) des Deutschen.
\end{abstract}

\section{Von Schemata (Bybee 1985) zu usage-based (Tomasello 1992, 2003) zu Konstruktionen (Goldberg 1995; Tomasello 2003; Bybee 2007)}

Ich möchte zunächst darlegen, in welchem historischen Kontext ich die gegenwärtige Diskussion zu den in der Überschrift zu diesem Abschnitt genannten

Dagmar Bittner, Leibniz-Zentrum Allgemeine Sprachwissenschaft 
linguistischen Modellen sehe: Unter dem Terminus usage-based bzw. construction grammar kommen aktuell sehr verschiedene grammatiktheoretische Strömungen miteinander in Berührung. Hintergrund dieses Zusammentreffens sind die in den letzten Dekaden immer deutlicher gewordenen Erklärungsgrenzen generativer Grammatiktheorien. ${ }^{1}$ Etwa seit Mitte der 1990er Jahre löst man mit der Feststellung, dass das Ziel einer vollständig und ausschließlich kompetenzorientierten Modellierung des sprachlichen Wissens nicht erreichbar ist, keine breite Front der Entrüstung mehr aus. Die Entstehung der heute schon fast wieder vergessenen Optimalitätstheorie war klares Indiz dafür, dass dies auch von Vertreterinnen und Vertretern der generativen Grammatik so gesehen wurde. Die Optimalitätstheorie ging über die idealisierte Sprecherkompetenz hinaus, indem hörerseitige Perzeptionsbedingungen als einzubeziehende Größen angesetzt wurden und darüber hinaus außersprachlich begründete Präferenz- und Markiertheitsprinzipien in die Modellierung des sprachlichen Wissens aufgenommen wurden. Man kann dies als einen ersten Brückenschlag der generativen Linguistik zu funktional-orientierten, d. h. sich mindestens bis auf Hermann Pauls Prinzipien der Sprachgeschichte zurückbeziehende Grammatik- und Sprachtheorien sehen. Schon vor Einführung der Optimalitätstheorie entstand im Spektrum der funktionalen Grammatik das Schema-Modell (Bybee 1985). Auch dieses Modell stellte den Versuch dar, Erklärungsgrenzen streng regelbasierter Grammatikmodelle zu überwinden, wobei hier - zumindest in seiner Rezeption in Deutschland/Europa eher Regelkonzepte funktional-strukturalistischer als solche generativ-strukturalistischer Grammatikmodelle ${ }^{2}$ hinterfragt wurden. Für die jüngere Zeit kann man wohl sagen, dass das Schema-Modell im usage-based-Modell (s. u.) aufgegangen ist. Zumindest wird dies von Bybee $(2007,2013)$ selbst so gesehen. Die sich ebenfalls in den 1990er Jahren etablierende Konstruktionsgrammatik (Goldberg 1995)

1 Für eine kritische Bestandsaufnahme aus den Reihen der generativen Grammatik selbst vgl. u. a. Koster (2017).

2 Die gegenwärtige Theorienlandschaft in der Linguistik ist nicht nur durch viele sich z. T. überlappende Positionen und Modelle gekennzeichnet, sondern auch dadurch, dass sehr unterschiedliche Modelle dieselben Termini benutzen. Benutzt man z. B. in einem Vortrag zum Spracherwerb den Terminus „funktional“, wird man von vielen Zuhörern dem generativen Modell zugeordnet und erntet Missverständnisse und böse Kommentare, wenn sich zeigt, dass man nicht von funktionalen Projektionen oder Kategorien spricht, sondern von inhaltlichkommunikativer Funktion. Ähnliches gilt für Termini wie „kognitiv“ oder „strukturalistisch“. Es ist mir immer schwergefallen, meine eigene theoretische Position zu benennen. Wenn es das gäbe, würde ich ,jakobsonistisch“ sagen und damit vor allem Jakobsons Primat der Bedeutung und seine auf Invarianten orientierte merkmalstheoretische Analytik meinen. Ich hoffe, mit „funktional-strukturalistisch“ terminologisch diese Zuordnung sowie die Abgrenzung von generativen und von gebrauchsorientierten Modellen zu treffen. 
ist das parallele Ablösekonzept aus der generativen Grammatik heraus. Ein gemeinsamer Nenner all dieser Entwicklungen ist das alte Problem der Modellierung von Regel und Ausnahme bzw. Kern und Peripherie im sprachlichen Wissen. Während sich das Schema-Modell seiner Herkunft gemäß vor allem mit der Modellierung morphologischer Strukturen befasst hat, betrachtet die Konstruktionsgrammatik ihrer Herkunft gemäß primär syntaktische Strukturen. In der weiteren Entwicklung wurden beide Modelle, Schema-Modell und Konstruktionsgrammatik, von ihren Vertretern als kompatibel und verwandt mit dem von Tomasello (1992) zur Erklärung des kindlichen Spracherwerbs propagierten usage-based-Modell gesehen, so wie auch Tomasello (2003) die Konstruktionsgrammatik in der Version von Goldberg als kompatibel mit dem usage-based-Modell sieht. Es fragt sich, welche theoretischen Annahmen beim Zusammentreffen von Konstruktionsgrammatik und Schema-/usage-based-Modell aufgegeben wurden. ${ }^{3}$

Je nach Ausgangspunkt der Theorieentwicklung auf der Skala ,generativ funktional' haben sich sowohl zahlreiche Facetten der Konstruktionsgrammatik (vgl. Hoffmann \& Trousdale 2013; Fischer \& Stefanowitsch 2006) als auch unterschiedlich strenge Facetten des Schema-/usage-based-Modells herausgebildet. Ich werde mich in der folgenden Diskussion auf die Facetten bzw. theoretischen Annahmen beziehen, die als vollständige Alternative nicht nur zur generativen Grammatik (wie das bei Goldberg und Tomasello der Fall ist), sondern zu einer lexembezogene Regeln annehmenden Grammatik überhaupt postuliert wurden (so verstehe ich z. B. Bybee 2013). Meine Frage ist, ob man tatsächlich auf lexembezogene Abstraktionen verzichten kann und warum man es sollte.

Zuvor aber der Blick auf Daten. Ich werde zunächst unter Rückgriff auf Bittner (2006) meine funktional-strukturalistische Analyse des frühen Erwerbs der definiten Artikel und Pronomen (der, die, das, den, dem) vorstellen und anschließend darlegen, aufgrund welcher Probleme aus meiner Sicht der Versuch einer konstruktionsgrammatischen Analyse dieses Erwerbsprozesses scheitert.

\section{Form und Bedeutung der relevanten definiten Artikel und Pronomen}

Definite Artikel und Pronomen, hier also D-Formen, sind im Deutschen bekanntlich der primäre Träger der Genus-Kasus-(und Numerus)-Merkmale einer defini-

3 Da es hier nicht um eine vollständige Aufarbeitung der Herausbildung der Konstruktionsgrammatik geht, bleiben zahlreiche andere Modellierungsvorschläge (z. B. duale Modelle und Hybridmodelle), die einen Anteil an der skizzierten Entwicklung gehabt haben, unerwähnt. 
ten Nominalphrase (DP). Obwohl die drei Merkmale fusioniert realisiert werden z. T. gestützt durch Merkmale an Nomen und Adjektiv -, sind sie sehr unterschiedlich in ihrer Funktionalität. Genus und Numerus stellen Informationen zur aktuell gewünschten mentalen Repräsentation des lexikalischen Konzepts, d. h. des Referenten der DP bereit (s. der Kranke, das Kranke, die Krankheit, die Kranken, die Krankheiten). Als nicht-paradigmatische Kategorie ist Genus allerdings bei den Simplizia eine inhärente Eigenschaft des Nomens und trägt, wie die Beispiele zeigen, erst in Interaktion mit Wortbildungsmitteln zur mentalen Repräsentation des Referenten bei. Kasus auf der anderen Seite hat nicht die mentale Repräsentation des Referenten zum Gegenstand, sondern die Repräsentation der Rolle bzw. des Status des Referenten im geschilderten Sachverhalt.

Soweit die funktional-strukturalistische Sicht auf die nominalen Kategorien (vgl. dazu auch Coseriu 1975; Jakobson 1936/1971; Leiss 1992; Bittner 2002). Will man den Erwerbsprozess bestimmen und erklären, der hinter dem Erscheinen der D-Formen in der kindlichen Sprachproduktion steht, ist es aus dieser Perspektive essentiell, die Funktion der nominalen Kategorien trotz oder gerade wegen ihrer fusionierten Realisierung auseinanderzuhalten. Dies wird in der Darstellung der Erwerbsdaten deutlich werden.

Die Annahme grammatischer Kategorien mit einem bestimmten Funktionsspektrum galt nach meinem Verständnis ursprünglich auch für das SchemaModell. Es wurden hier zwar andere Repräsentationen der morphologischen Formen und der Relationen zwischen den Formen angenommen, es wurde aber nicht auf die Bestimmung grammatischer Kategorien, ihrer Funktionen sowie der Relationen zwischen und innerhalb kategorialer Domänen verzichtet (vgl. z. B. Bybee et al. 1994 zum System der Aspekt-Tempus-Modus-Kategorien (sowie schon Bybee 1985) oder Köpcke 1993 zur Genus-Numerus-Relation). In einem gebrauchsorientierten Konzept wie der Konstruktionsgrammatik dagegen existieren nicht-formelle Kategorien streng genommen gar nicht (also grammatische Kategorien wie Numerus/Kasus, die eine Funktion und nicht primär eine sprachliche Einheit wie NP/DP repräsentieren). Sie sind - wenn man so will - in den Konstruktionsbedeutungen aufgegangen. Für den Sprachgebrauch ist die innersprachliche Organisationsebene uninteressant. Es wird schließlich nicht über sprachliche Strukturen kommuniziert (sich unterhalten). Gebrauchsorientierte Konstruktionsbedeutungen müssten konsequenterweise die durch eine Konstruktion transportierte Information ohne Bezug auf metasprachliche grammatische Termini wiedergeben. ${ }^{4}$

4 Hier liegt möglicherweise auch die Quelle für die Aufspaltung der Konstruktionsgrammatik in sehr unterschiedliche Richtungen. Das Primat der Konstruktionsbedeutung verlagert die 
Bevor ich nun endlich zu den Erwerbsdaten komme, noch ein traditioneller Blick auf die Form-Funktions-Verteilung der D-Formen. Abbildung 1 gibt das bekannte Paradigma der pronominalen Flexion definiter Artikel/Pronomen wieder.

\begin{tabular}{lllll}
\hline & \multicolumn{3}{l}{ Singular } & Plural \\
\cline { 2 - 4 } & Fem. & Mask. & Neutr. & \\
\hline Nom. & die & der & das & die \\
Gen. & der & des(sen) & des(sen) & der(en) \\
Dat. & der & dem & dem & den(en) \\
Akk. & die & den & das & die \\
\hline
\end{tabular}

Abb. 1: Das Paradigma der Genus-, Numerus-, Kasusformen definiter Artikel und Pronomen.

Die Analyse der Erwerbsdaten wird sich nur auf die Singularformen im Nominativ, Akkusativ und Dativ beziehen (fett gedruckte Formen). Genitive generell wie auch die komplexeren Pronomen im Plural sind in den frühen Spontansprachdaten nicht belegt. Die Formverschiedenheiten bei Artikeln und Pronomen in Genitiv und Plural können daher hier ausgeblendet werden.

Die zwar bekannte aber wichtige Feststellung ist, dass ein hoher Grad an Synkretismus vorliegt. Alle fünf D-Formen, die in den frühen Spontandaten auftreten (die, der, das, den, dem) kommen in mehreren kategorialen Positionen des Paradigmas vor, d. h. oberflächenbasiert betrachtet in mehreren kategorial unterschiedlichen grammatischen Positionen. Aus der Oberflächenperspektive müssten also für alle fünf Formen mehrere Bedeutungen und/oder Funktionen angesetzt werden. ${ }^{5}$

Strukturalistische Analysen sehen einige oder auch alle der Synkretismen als Unterspezifikation bzw. Neutralisierung von auf der sprachlichen Oberfläche vor-

\footnotetext{
Grammatikbeschreibung auf eine von der traditionellen Grammatikschreibung völlig verschiedene Ebene. Es ist nicht einfach eine Verlagerung der Gewichtung auf die Bedeutungsseite sprachlicher Ausdrücke. Das sprachliche Wissen soll als System (?) der kommunikativen Bedeutung von Konstruktionen repräsentiert werden. Das verlangt im Grunde eine völlig andere Terminologie als die traditionelle auf grammatische Kategorien bezogene Terminologie. Diese steht bisher nicht zur Verfügung, u. a. deshalb, weil es - wie ich auch im Folgenden zu zeigen versuche - schwierig ist $\mathrm{zu}$ entscheiden, was als kommunikativ bedeutungstragende Konstruktion anzunehmen ist. Je kleinteiliger man hier wird, umso näher ist man an den sprachlichen Einzelzeichen und ihren (traditionell beschriebenen) Bedeutungen.

5 Funktion und Bedeutung von grammatischen Formen sind für mich weitgehend das gleiche. Ich werde im Folgenden nur noch von Funktion(en) sprechen und dabei (auch) die Bedeutung(en) meinen, die durch die jeweiligen Strukturen transportiert werden.
} 
liegenden kategorialen Unterschieden an (vgl. Bierwisch 1967; Bittner 2002; Müller 2003 und die weitere dort genannte Literatur). Durch die D-Formen nicht ausgedrückte kategoriale Unterschiede werden durch andere Mittel wie zum Beispiel Wortstellung, Prosodie (Das Pferd bedrängt das Kamel.) oder Subjekt-Verb-Kongruenz (die ist groß. vs. die sind groß.) realisiert. Die Unterspezifikationsanalysen legen nahe, dass keine zufälligen Formgleichheiten vorliegen. Synkretismen motivieren sich vielmehr aus Gemeinsamkeiten der beteiligten Kategorien. Sie lassen schlicht spezifischere Merkmale komplexerer Kategorien unausgedrückt.

\section{Der Erwerb der D-Formen bis zum Alter von 3;0}

Die Verwendung von D-Formen beginnt bei deutsch-monolingual aufwachsenden Kindern typischerweise um den 2. Geburtstag, also mit ca. 24 Monaten. Es werden von Beginn an deutlich mehr „richtige“ als „falsche“ D-Formen produziert. In den Daten von Simone, einem der untersuchten Kinder, finden sich zwischen 1;11 und 2;4 in nur knapp $2 \%$ aller Äußerungen, die eigenständig produzierte lexikalische Einheiten enthalten, nicht-zielsprachliche D-Formen (Bittner 2006). Dies scheint auf den ersten Blick so gering, dass man diese „Fehler“ als vernachlässigbar betrachten möchte. Doch schon aus empirischen Gründen und erst recht aus theoretischen Gründen sind sie nicht zu vernachlässigen. Die nicht-zielsprachlichen Formen sind die einzige Möglichkeit festzustellen, ob Kinder Generalisierungen über Form-Funktions-Zuordnungen in ihrer Grammatik etabliert haben. In Modellen, die lexembezogene Generalisierungen ${ }^{6}$ vorsehen, wären dies Muster, Schemata oder Regeln über zulässige Äußerungskontexte der D-Formen. In konstruktionsgrammatischen Modellen sollten es kind- oder altersspezifische Bedeutungen der Konstruktionen sein, in denen die D-Formen auftreten.

In Bittner (2006) wurde die Hypothese aufgestellt, dass im ersten Jahr der Verwendung von D-Formen in der kindlichen Grammatik Generalisierungen für singularisches die, der und den etabliert werden. Alle drei Formen werden mit der Funktion ,definit‘ assoziiert, was eine Abgrenzung von anderen adnominalen Begleitern, die in derselben Position auftreten können, wie indefinitem Artikel, Negationsartikel oder Quantoren beinhaltet. Die Formen der und den werden zu-

\footnotetext{
6 Der Terminus „lexembezogen“ steht für ein grammatiktheoretisches Herangehen, das u. a. die Erfassung der Einsetzungsbedingungen sprachlicher Einzelzeichen (Lexeme) und der darauf aufbauenden Kategorisierung der Einzelzeichen $\mathrm{zu}$ syntaktisch relevanten Klassen anstrebt.
} 
sätzlich mit Funktionen assoziiert, die den Argumentstatus der DP spezifizieren. ${ }^{7}$ Diese Form-Funktions-Zuordnungen ergeben das in Tabelle 2 veranschaulichte uniforme Paradigma der D-Formen. Es gibt in dieser Erwerbsphase keine Aufsplittung in mehrere singularische Paradigmen, weil Genus noch nicht in der Funktionszuweisung zu den D-Formen berücksichtigt wird.

\begin{tabular}{lll}
\hline D-Form & Generelle Funktion & Spezifische Funktion \\
\hline die & definit & \\
der & definit & Subjekt (Agens) \\
den & definit & Objekt (Patiens) \\
\hline
\end{tabular}

Abb. 2: Das uniforme Singular-Paradigma in der Grammatik von ca. $2 ; 6-3 ; 0$.

Die Hypothese über das Vorliegen dieser Form-Funktions-Zuordnungen in der Grammatik von Kindern im Alter von ca. 2;6-3;0 ergibt sich aus der Analyse der nicht-zielsprachlich verwendeten D-Formen. Die Verteilung der entsprechenden DFormen in den Spontansprachdaten von drei Kindern (Simone, Katrin, Anna) ist in Abbildung 3 und 4 wiedergegeben. ${ }^{8}$ Während nicht-zielsprachliche D-Formen in der Erwerbsliteratur nahezu ausschließlich im Hinblick auf das Genus des Bezugsnomens beurteilt wurden, bezieht diese Analyse auch die Kasuskontexte ein (vgl. auch Wegener 1995 und 2000 mit parallelen Ergebnissen zum L2-Erwerb).

Die Daten legen die Unterscheidung von 2 Phasen in der Verwendung der D-Formen im 3. Lebensjahr nahe. Phase I (Abb. 3) umfasst das Alter von 1;102;4 bei Simone, 1;11-2,2 bei Anna und von 2;2 bei Katrin. Phase II (Abb. 4) umfasst das Alter von 2;5-2,11 bei Simone, von 2;3-2;10 bei Anna und von 2;3-2,6 bei Katrin. Das spätere Einsetzen der Analysen bei Katrin sowie der frühere Schluss bei Anna und Katrin resultieren aus der Dauer der Datenerhebung bei beiden Kindern. Abbildung 3 und 4 präsentieren die Anzahl der in den Daten

7 Welche Merkmale zur Differenzierung des Argumentstatus der DPs in der Grammatik des Kindes etabliert werden, ist noch offen. $\mathrm{Zu}$ ihrer Darstellung verwende ich provisorisch die Satzgliedtermini. In Bittner (2006) wurden sie als Kasusmerkmale betrachtet.

8 Die Analyse der Daten von Simone wurde in Bittner (2006) vorgestellt. Die Analyse der Daten von Katrin und Anna wurde bisher nicht publiziert (Bittner 2004 poster). Die Längsschnittkorpora von Simone sind auf der CHILDES-Datenbank zugänglich (https://childes.talk bank.org/browser/index.php?url=Germany). Die Daten von Katrin entstammen dem zu Beginn der 1990er Jahre durchgeführten DFG-Projekt "Die Entwicklung der regulären und irregulären Flexionsmorphologie des Deutschen” unter der Leitung von H. Clahsen. Das Korpus von Anna wurde von mir, D. Bittner, Mitte der 1990er Jahre erhoben. Es ist auf Anfrage zugänglich. 
belegten nicht-zielsprachlichen Verwendungen von D-Formen sortiert nach Genus des Bezugsnomens (vertikal) und Kasuskontext, in dem die DP verwendet wurde (horizontal).

\begin{tabular}{|c|c|c|c|c|c|c|c|c|c|c|}
\hline \multirow{2}{*}{$\begin{array}{l}\text { Kasuskontext } \\
\text { produzierte } \\
\text { Form }\end{array}$} & \multicolumn{3}{|c|}{ Nom } & \multicolumn{4}{|c|}{ Akk } & \multicolumn{2}{|r|}{ Dat } & \multirow[t]{2}{*}{ total } \\
\hline & die & der & den das & die & der & den & das & die & der den das & \\
\hline Bez.nom. Fem. & & 10 & & & & 3 & & 3 & & 16 \\
\hline Bez.nom. Msk. & 6 & & 1 & 1 & 3 & & 3 & 1 & 2 & 17 \\
\hline Bez.nom. Ntr. & 2 & 18 & & 3 & & 7 & & & & 30 \\
\hline total & 8 & 28 & 1 & 4 & 3 & 10 & 3 & 4 & 2 & 63 \\
\hline
\end{tabular}

Abb. 3: Nicht-zielsprachliche D-Formen in Phase I.

\begin{tabular}{|c|c|c|c|c|c|c|c|c|c|c|c|c|}
\hline \multirow{2}{*}{$\begin{array}{l}\text { Kasuskontext } \\
\text { produzierte } \\
\text { Form }\end{array}$} & \multicolumn{4}{|c|}{ Nom } & \multicolumn{4}{|c|}{ Akk } & \multicolumn{3}{|c|}{ Dat } & \multirow[t]{2}{*}{ total } \\
\hline & die & der & den & das & die & der & den & das & die & der & den das & \\
\hline Bez.nom. Fem. & & 17 & & 5 & & 1 & 11 & 1 & 11 & & & 46 \\
\hline Bez.nom. Msk. & 20 & & 4 & 3 & 10 & 4 & & 2 & & 3 & 11 & 57 \\
\hline Bez.nom. Ntr. & 7 & 15 & 2 & & 5 & 1 & 5 & & 3 & & 8 & 46 \\
\hline total & 27 & 32 & 6 & 8 & 15 & 6 & 16 & 3 & 14 & 3 & 19 & 149 \\
\hline
\end{tabular}

Abb. 4: Nicht-zielsprachliche D-Formen in Phase II.

In Phase I dominiert die nicht-zielsprachliche Verwendung von der in Nominativkontexten. Im Akkusativ zeichnet sich eine Dominanz nicht-zielsprachlicher den-Verwendungen ab. Die geringe Anzahl der Belege in Dativkontexten resultiert aus der noch relativ einfachen Äußerungsstruktur in dieser Erwerbsphase. ${ }^{9}$ Der Übergang zu Phase II wurde am Einsetzen einer verstärkten nicht-zielsprachlichen Verwendung von die in allen drei Kasuskontexten festgemacht. Nichtzielsprachliches der erscheint weiter primär in Nominativ- und nichtzielsprachliches den in Akkusativkontexten, wobei den jetzt zusätzlich auch in Dativkontexten dominiert, d. h. den erscheint in beiden Objektkontexten. ${ }^{10}$

9 Phase I fällt in der syntaktischen Entwicklung mit dem Übergang zu Mehrwortäußerungen zusammen. Bei Anna zum Beispiel liegt mit 2;2 eine mittlere Äußerungslänge von 2,5 Wörtern vor. Das bedeutet, dass sie nur eine begrenzte Anzahl von Äußerungen produziert, die mehr als ein oder zwei referentielle Ausdrücke enthalten, mehrheitlich also nominativische und/ oder akkusativische Ausdrücke.

10 Im Hintergrund dieser im Spracherwerb länger anhaltenden Übergeneralisierung/Neutralisierung - s. auch die von Grießhaber (i. d. B.) aufgezeigten hohen Fehlerraten in der Dativflexion 
In Phase II finden sich auch - hier nicht berücksichtigte - nicht-zielsprachliche Verwendungen von die im Dat.Pl. (u. a. Simone 2;8.15 mit die Manns hier; Anna 2;9.22 bei die katzen) und den im Akk.Pl. (u. a. Simone 2;9.28 nicht den haare). Sie entsprechen der angenommenen Funktionszuordnung für diese Formen. Nichtzielsprachliche Verwendungen von das sind im gesamten Zeitraum in allen Kasuskontexten marginal. ${ }^{11}$

Bei allen drei Kindern zeigen sich die gleichen Präferenzen in der nichtzielsprachlichen Verwendung von die, der und den. Dies bestätigt die in Bittner (2006) anhand der Daten von Simone aufgestellte Hypothese zur Funktionszuordnung von die, der, den in der kindlichen Grammatik im Alter von ca. 2;6 bis 3;0 (s. Abb. 2). ${ }^{12}$

\section{Argumente für die vorgestellte lexembezogene Analyse}

Die vorgestellte Analyse ist lexembezogen. In der aus dieser Perspektive erfolgten Forschung wird angenommen, dass D-Formen spätestens bis zum Alter von 2;0 als sprachliche Zeichen aus dem Sprachstrom extrahiert werden (Clahsen 1984; Tracy 1986; Müller 1994; Bittner 1998). Das beinhaltet notwendig die Annahme, dass die Formen mit eigenständigen Funktionsmerkmalen (Bedeutungen) assoziiert wurden. Ich nehme an, dass der Hypothesenraum für die Form-Funktions-Zuordnung für die aus dem Input ermittelten D-Formen im Erwerbsprozess schrittweise beschränkt wird. Die Beschränkungsrichtung ergibt sich für die Kinder aus der im sprachlichen Input festgestellten Verteilung der D-Formen auf nominale Ausdrücke (bzw. in der frühen Grammatik „konkrete“ Ausdrücke im Gegensatz zu relationalen Ausdrücken) und den im Input vorgefundenen Alternativen zur Verwendung von D-Formen wie indefiniten Artikeln, Possessiv- und Negationsartikeln, m. a. W. aus den syntagmatischen und paradigmatischen Oppositionen, in denen D-Formen auftreten.

im L2-Erwerb - steht die im Beitrag von Radden (i.d.B.) thematisierte stärkere Spezifik des Dativ als Objektkasus gegenüber dem Akkusativ.

11 Die Analyse der das-Formen ist nur bedingt möglich. Gerade in den frühen Daten gibt es viele Verwendungen, bei denen nicht entscheidbar ist, ob das eine Verschmelzung von das + ist darstellt, vgl. Simone 2;2.20 das Elefant das ist $+/ /$ (+// bedeutet: Unterbrechung durch Kommunikationspartner).

12 Hypothesen darüber, in welchen Schritten Kinder von diesem uniformen Paradigma zu den nach Genus differenzierten Paradigmen der Erwachsenensprache kommen, sind in Bittner (2006) anhand der Simone-Daten aus dem 4. Lebensjahr dargelegt. 
Im Folgenden fasse ich über die obige Analyse hinausgehende Argumente zusammen, die m. E. dafür sprechen, dass eine solche lexembezogene Analyse den Anspruch erheben kann, den Erwerbsprozess der D-Formen bis zum Alter von 3;0 erklärungsadäquat zu erfassen - wenn eventuell auch nicht genau in der hier vorgestellten Version. Im Kern geht es um die Frage, wodurch der Hypothesenraum für die Erschließung der Form-Funktions-Zuordnung für die DFormen begrenzt wird.

(1) Es gilt als gesichert, dass die Segmentierung des Sprachstroms im Erwerbsprozess über satzartige Äußerungen zu Konstituenten zu Wörtern verläuft (vgl. Weinert \& Grimm 2012). Es trifft daher zu, dass zumindest die als Artikel verwendeten D-Formen zuerst in Verbindung mit Nomen wahrgenommen werden. Sehr wahrscheinlich kann die Bindung an Nomen auch für die D-Pronomen angenommen werden, da der Bezug auf das Nomen in der frühen Interaktion von/mit Kindern kontextuell abgesichert wird. Daraus folgt, dass der Hypothesenraum für die Funktionsbestimmung von D-Formen von Beginn an auf nominale Argumente, ihre internen (formalen) und externen (syntaktischen) Eigenschaften, begrenzt ist. ${ }^{13}$

(2) Trotz des vermutlich bereits im 1. Lebensjahr etablierten Wissens über die Zugehörigkeit von D-Formen zu nominalen Ausdrücken (zur sprachlichen Entwicklung im 1. Lebensjahr vgl. u. a. Höhle 2015) beginnt ihre Produktion etwa ein Jahr später als die Produktion von Nomen. Im Alter von 20 Monaten enthält das produktive Lexikon deutsch-monolingual aufwachsender Kinder durchschnittlich 80 verschiedene Nomen (Suchodeletz 2010). In den Daten von Simone sind bis 2;1 (26 Monate) über 60 verschiedene Nomen mit definitem Artikel belegt. Fast alle Nomen werden zuerst als sogenannte bare nouns produziert. $\mathrm{Zu}$ Beginn der Produktion von nominalen Ausdrücken gibt es somit keine bzw. nur sehr beschränkt unanalysierte holistische DP-Konstruktionen. DPs werden bereits vor dem Beginn der Determiniererproduktion intern analysiert. Das gilt auch, wenn die im frühen Input hochfrequenten

13 Diese Annahme bedeutet u.a., dass die Form-Funktions-Zuordnung für deiktisches und auf Sachverhalte bezogenes das wie in das da oder das hast Du fein gemacht kein Bestandteil des Erwerbsprozesses der Genus-Kasus-Funktionen der D-Formen ist, denn es existiert in dieser Verwendung kein Bezugsnomen. M.E. wird die primär deiktische Verwendung von das vor dem Erwerb der Genus-Kasus-Funktionen erworben und anhand eines Merkmals wie ,unspezifisch deiktisch“ von dem der D-Formen mit Bezugsnomen abgegrenzt (Bittner 2007). Einen Hinweis auf die Existenz relevanter Unterschiede gibt folgendes Beispielpaar: Kind zeigt auf etwas und sagt: das - mögliche Reaktion der/des Erwachsenen: ah, ein/der Ball liegt da vs. Kind zeigt auf etwas und sagt: den - mögliche Reaktion der/des Erwachsenen: ah, ${ }^{*}$ einen Ball/den Ball willst Du haben. 
Eigennamen regelmäßig mit einem definiten Artikel verbunden werden, wie die Daten von Simone zeigen. Zudem gibt es Kinder, wie z. B. Anna, die parallel zum Erscheinen von DPs intensiv D-Pronomen produzieren. Das Hinzufügen eines Determinierers - egal ob definit oder indefinit - $\mathrm{zu}$ einem Nomen ist ein gezielter Akt der Spezifizierung des nominalen Ausdrucks (wie immer ,Nomen' bzw. ,nominal' in der aktuellen Grammatik des Kindes auch etabliert ist). Mit anderen Worten, es ist der Determinierer, dem in der kindlichen Grammatik eine bestimmte Funktion (Bedeutung!) zugewiesen worden ist. Die Konstruktion bekommt diese Funktion via Determinierer. ${ }^{14}$

(3) Die Verwendung von D-Formen in "falschen“ determinativen Kontexten, also in Kontexten, in denen zielsprachlich andere Determinierer oder Pronomen stehen, ist bisher in der Literatur zum Erstspracherwerb des Deutschen nicht berichtet worden. In den dieser Studie zugrundeliegenden Daten findet sich ebenfalls kein Hinweis darauf. Die oben besprochenen nicht-zielsprachlichen Verwendungen von D-Formen sowie die bei vielen Kindern anfänglich zu beobachtende Verwendung von reduzierten D-Formen (zumeist de) erfolgt nur in Kontexten, in denen auch zielsprachlich DFormen stehen. Unmittelbar vor oder zeitgleich mit den D-Formen beginnt die Produktion von Possessivartikeln (mein, meine) und indefiniten Artikeln (ein, eine).

Aus dem unter (2) und (3) Dargelegten lässt sich schlussfolgern, dass D-Formen von Beginn ihrer Verwendung an funktional von anderen Determinierern, die in derselben syntaktischen Position auftreten können, durch ihre Assoziation mit dem Merkmal ,definit' (oder auch ,sprachlich/situativ bekannt`) abgegrenzt werden. Der Hypothesenraum für die weitere, interne Differenzierung der D-Formen (s. Abschnitt 3) wird damit auf die Kontexte von DPs beschränkt. Die beschriebene Form-Funktionszuordnung ist Teil einer kontinuierlichen Einschränkung des Hypothesenraumes, die letztendlich zur funktionalen Spezifizierung des in 2 beschriebenen Formeninventars und im Anschluss daran zu den Genus-Numerus-basierten Paradigmen der D-Formen führt.

14 Wie in einer der Reviews angemerkt wurde, könnte es sein, dass der Erwerb der Funktion der DP-Konstruktion noch andauert, wenn die Produktion der Nomen beginnt. Man müsste unter diesem Primat der Konstruktion aber erklären, warum aus dem Inputangebot zuerst gerade die bare-noun-Konstruktion ausgewählt wird und warum sie auch in den Kontexten von Determiniererkonstruktionen verwendet wird. Aus der Inputfrequenz lässt sich dies nicht ableiten. 


\section{Versuch einer konstruktionsgrammatischen Analyse}

Betrachtet man Konstruktionen als Ausgangsbasis für die kindliche Analyse des Inputs im Hinblick auf die Verwendung von D-Formen, ist die erste Frage, welche(s) ist/sind die relevante(n) Konstruktion(en)? Satz oder Konstituente oder doch nur die Nominalgruppe? Man kann wohl davon ausgehen, dass alle diese strukturellen Einheiten in der (passiven) Grammatik des zweijährigen Kindes vorliegen (Höhle 2015). Um sie als Konstruktion im Sinne der Konstruktionsgrammatik zu etablieren, müssen ihnen aber Bedeutungen zugeordnet werden. Geht man von oberflächennahen Konstruktionsbedeutungen aus, was man m.E. muss, wenn Konstruktionen als holistische Strukturen aufgefasst werden (so sieht es m. E. auch die radikale Konstruktionsgrammatik von Croft 2001), sind diese aber eben vielfältig und es ist bisher kein Modell vorgestellt worden, das beschreibt und geschweige denn voraussagt, welche Konstruktionen wann erworben werden. Bisher lag der Fokus konstruktionsgrammatischer Erwerbsstudien auf satzwertigen Konstruktionen (Tomasello 1992; Goldberg 2006). Diese sind aber definitiv ein viel zu weiter, weil im Grunde ja unbeschränkter Hypothesenraum für die Erschließung der Verwendungsbedingungen (traditionell gesprochen: der Form-Funktions-Zuordnung) von D-Formen bzw. D-Form-haltigen Konstruktionen. Abgesehen davon liegt das an der Produktion festgemachte Erwerbsalter für abstrakte satzwertige Konstruktionen mit offenen Slots für bestimmte Argumente deutlich nach dem 2. Geburtstag, d. h. nach dem Einsetzen der nicht-zielsprachlichen Verwendung von D-Formen.

Aber auch wenn man für die hier interessierenden nominalen Strukturen einen Erwerbseinstieg bei Konstruktionen unterhalb der Satzebene, konkret auf Konstituentenebene, in Betracht zieht, wird es nicht wirklich einfacher. Die kritische Frage ist: Was fließt alles in eine Konstruktionsbedeutung ein? Im Grunde wie oben schon gesagt - doch alles, wenn Konstruktionen oberflächenbezogene Strukturen sind. Es kann nichts hergeleitet werden, wenn es keine Hierarchien und Abhängigkeiten zwischen Konstruktionen gibt, wenn jede konventionalisierte Konstruktion letztlich ein Idiom für sich ist. Konstituenten, speziell Argumente und Adjunkte, mögen im Hinblick auf ihre semantischen Rollen als Konstruktionen klassifizierbar sein. Ihre strukturelle Ausprägung wird durch diese Klassifizierung aber kaum beschränkt (vgl. Ágel \& Höllein i.d.B.). Eine Präpositionalgruppe ist eine andere Konstruktion als eine definite oder eine indefinite Nominalgruppe, eine singularische Nominalgruppe eine andere als eine pluralische als eine generische . . . . Für die D-Formen des Deutschen lässt sich auch nicht sagen, dass der Hypothesenraum auf solche Konstruktionen und 
Konstruktionsbedeutungen beschränkt werden kann, die referentielle Ausdrücke mit einem Nomen als lexikalischem Kern bilden. Zum Analysesample gehören auch D-Pronomen und referentielle wie nicht referentielle Präpositionalgruppen (mit der (Oma), an dem Tag usw.). Zumindest ist bisher kein Unterschied im Erwerb der Form-Funktions-Zuordnung (traditionell gesprochen: der Paradigmenentwicklung) der jeweiligen D-Formen festgestellt worden. Welche Vervielfachung an Konstruktionsbedeutungen dies theoretisch bedeutet, ist schon kaum noch vorstellbar. Ganz offensichtlich lässt sich der Hypothesenraum weder durch oberflächennahe Konstruktionsbedeutungen noch durch die Eigenschaft ,referentieller Ausdruck/Verweis‘ beschränken. ${ }^{15}$

Es lässt sich einwenden, dass Konstruktionsbedeutungen ein Ergebnis des Erwerbsprozesses sind und im 3. Lebensjahr noch kein aufgefächertes Netz von Konstruktionsbedeutungen vorliegt. Sind Konstruktionen also für die frühe Differenzierung von zum Beispiel der und den irrelevant? Zur Erinnerung: Nichtzielsprachliches der erscheint fast ausschließlich im Subjekt, also im Nominativ Singular, während nichtzielsprachliches den fast ausschließlich im Objekt, also im Akkusativ/Dativ Singular erscheint. Subjekt und Objekt sind strukturelle Funktionen, die durch Argumente (Konstruktionen?) verschiedener Art realisiert werden. Damit kommt wieder die Frage ins Spiel, welche Konstruktionen (Form-Bedeutungs-Paare) als Erwerbsbasis anzusetzen sind. An diesem Punkt waren wir schon.

Hilft eine andere zentrale Annahme von konstruktionsgrammatischen und usage-based-Konzepten zum Spracherwerb weiter? Tomasellos (1992) verb island hypothesis folgend wird angenommen, dass der Erwerb von Konstruktionen

15 Ein Reviewer fragte, ob das Argument der Vervielfachung der Konstruktionsbedeutungen wirklich einen Ausschlussgrund für die Interpretierbarkeit im konstruktionsgrammatischen Ansatz darstellt. M. E. ist das der Fall, solange Konstruktionsbedeutungen und ihre strukturelle Erzeugung nicht als systematisch aufeinander bezogen und damit letztlich auch beschränkt erfasst sind. Ohne einen innersystematischen Bezug aufeinander bleiben Konstruktionen und Konstruktionsbedeutungen eine beliebig vervielfachbare Liste. Dies machen Ágel und Höllein (i.d.B.) in Bezug auf semantische Rollen sehr deutlich. Ihre Warnung vor einer „Verwechslung von Bedeutung und Bezeichnung, von Einzelsprache und Welt“ und davor „anstelle der Sprache die Welt zu analysieren“ ist m. E. höchst relevant. Strukturalistische Modelle gehen mit der Annahme von Einsetzungsbedingungen auf Einzellexemebene, Lexemklassenebene und schließlich auf Konstituenten- und Illokutionsebene von vornherein von einer systematischen Beschränkung der sprachlichen Mittel und einem innersystematischen Bezug zwischen ihnen aus. In diesem Sinne beschränken Determinierer mit ihren Einzellexemeigenschaften den Bedeutungsrahmen von nominalen Ausdrücken unterschiedlichster Art. Jede Determiniererklasse tut dies in systematischer Relation zu den anderen in derselben Sprache existierenden Determiniererklassen. 
Einzelwort-bezogen erfolgt und erst Generalisierungen über viele solche Einzelwortstrukturen zu abstrakteren Konstruktionen führen. Auch für den Determinierererwerb wurde postuliert, dass er sich anfangs bei jedem Nomen individuell vollzieht (Pine et al. 2013 zum Englischen). Ist der Hypothesenraum also durch das Bezugsnomen definiert, durch die Konstituentenvarianten, in denen ein Bezugsnomen im Input auftritt? Geht das ohne Bezug auf die Funktion der Konstituenten in einer komplexeren Konstruktion? Das Kind kann zum Beispiel feststellen, dass die Nomen Puppe und Uhr im Input mit den D-Formen die und der verbunden werden. Die nichtzielsprachliche Verwendung von der Puppe im Nominativ Singular (Simone 2;01.12 eine Knetpuppe auf einen Stuhl legend: komm \# der puppe die geht da (r)auf) kann unter dieser Voraussetzung noch als Unsicherheit über die Verwendungskontexte von die und der in Verbindung mit Puppe oder den aktuellen Status der Konstituente erklärt werden. Die Verwendung von den Uhr (Simone 2;00.03: den - den uhr habe - den uhr habe) ist unter der Beschränkung auf das Bezugsnomen aber nicht erklärbar. Sie erscheint schlicht als zufälliger Ausreißer.

Wenn das Bezugsnomen den Hypothesenraum für den Erwerb der D-Formen begrenzt, sollten nichtzielsprachliche Verwendungen bevorzugt die D-Form aufweisen, mit der das Nomen am häufigsten im Input anzutreffen ist. Ganz sicher sind dies die Nominativ- und Akkusativformen. Es wäre also zu erwarten, dass nichtzielsprachliche Verwendungen bei den Feminina die, bei Maskulina der und den und bei Neutra das aufweisen. Dies ist aber eben nicht das Muster, das sich in den Daten der drei untersuchten Kinder zeigt. Selbst wenn man annimmt, dass auch Präferenzen und Beschränkungen nominaler Ausdrücke für bestimmte Determinierer (z. B. weniger Artikelsetzungen und keine indefiniten Artikel bei Massennomen) und bestimmte Argument- bzw. Adjunktpositionen berücksichtigt werden (z. B. das gegenüber Maskulina seltenere Auftreten von Feminina und Neutra im Nominativ), ist weder das überindividuelle Auftreten von der im Nominativ Singular noch das von den im Akkusativ/Dativ Singular der Feminina und Neutra zu erklären und auch nicht das Auftreten von die in fast allen nicht-zielsprachlichen Kontexten. Ganz offensichtlich wird der Hypothesenraum mindestens parallel zum Bezugswort durch äußerungsstrukturelle Kontexte begrenzt. Es werden also (wie auch immer in ihrer Funktion bestimmte) Subjektkontexte von Objektkontexten unterschieden und Bedingungen für das Auftreten der verschiedenen Begleiter des Nomens in diesen Kontexten erschlossen. Schließlich, neben den Artikelverwendungen in Phase I, treten sehr frequent pronominal deiktische Verwendungen der D-Formen auf - im Fall von den stehen bei Simone zum Beispiel 30 pronominale Verwendungen 13 Artikelverwendungen gegenüber (ohne Dominanz eines bestimmten Nomens) - und es treten zusätzlich auch Verwendungen in Präpositionalphrasen (3 bei Simone) und im Plural auf. Es ist daher 
m. E. plausibler anzunehmen, dass in der Grammatik des Kindes eher eine lexembezogene Bedeutungszuweisung wie [den = Verwendung in „nominalen“ Ausdrücken zur Markierung von „Objekt“status] als eine konstruktionsbezogene wie [den $+\mathrm{N}=$ Objekt] etabliert wurde. Letzteres würde $\mathrm{u}$. a. auch die Annahme weiterer Konstruktionsbedeutungen für den enthaltende Konstruktionen in der Grammatik des Kindes erfordern.

Wie überzeugend ist das Argument für einen Einzelwort-basierten (,island“) Erwerb in Bezug auf Determinierer? Bedeutet eine wenig frequente Verwendung oder auch das Fehlen (d.h. das Nicht-belegt-Sein) bestimmter DeterminiererNomen-Verbindungen in den typischerweise untersuchten Daten mit häuslicher Eltern-Kind-Kommunikation, dass die jeweiligen Formen nicht erworben sind? Muss man nicht einbeziehen, welche Wahrscheinlichkeiten für das Verwenden bestimmter Strukturen in bestimmten Argumentpositionen und in bestimmten Kontexten bestehen? Kinder sind in der Eltern-Kind-Interaktion in einer anderen Diskursposition als die Erwachsenen. Sie geben den Erwachsenen eher selten Erklärungen und Anweisungen, während es umgekehrt durchaus anders ist. Dies könnte sich z. B. so auswirken, dass Kinder in solchen Kommunikationssituationen weniger Präpositionalphrasen und Adverbiale produzieren, einige davon vielleicht gar nicht (z. B. etwas wie: auf die Vase (achten/aufpassen)), obwohl sie sie durchaus beherrschen. ${ }^{16}$ Ebenso könnte es sein, dass jüngere Kinder in dieser Gesprächskonstellation vergleichsweise seltener neue Objekte (Topiks) in den Diskurs einführen als die Erwachsenen. Das könnte bewirken, dass sie weniger indefinite Nominalgruppen produzieren als die Erwachsenen, obwohl sie sie durchaus beherrschen. ${ }^{17}$ Die Gebrauchsfrequenz von Einzelwörtern und Konstruktionen in der kindgerichteten Sprache ist unbestritten ein wichtiger Faktor im Erwerbsverlauf. Auch die generative Grammatik hat den Input nicht als verzichtbar angesehen, sondern als Auslöser für die richtige Parametersetzung. Und natürlich werden über Frequenzen Gebrauchsmuster und -erwartungen geformt, anders lässt sich der niedrige Prozentsatz nichtzielsprachlicher D-Form-Verwendungen gar nicht erklären. Die von Beginn an zu beobachtenden nichtzielsprachlichen Instanzen zeigen aber, dass die Analyse ihrer Verwendungsbedingungen deutlich

16 Entsprechende Beispiele aus dem Bereich der Wortbildung zeigt Mattes (2018) auf: In der von ihr untersuchten Eltern-Kind-Kommunikation finden sich bis zum Alter von ca. 7 Jahren nur sehr vereinzelte Belege für nominale Ableitungen mit -erei und ge- - -e (Meckerei, Rennerei, Gelaufe, Getobe) bei den Kindern. In den Kind-Kind-Rollenspielen ihrer beiden Töchter im entsprechenden Alter beobachtet sie diese aber regelmäßig.

17 Dieser Aspekt wurde zum Beispiel von Pine et al. (2013) in ihrer Untersuchung zur Verwendung von a/an und the in der Eltern-Kind-Kommunikation nicht berücksichtigt (so wie generell keine funktionalen Unterschiede von indefiniten und definiten Artikeln). 
vor dem Produktionsbeginn einsetzt und der Hypothesenraum dafür deutlich mehr umfasst als Frequenzverhältnisse beim Bezugsnomen oder auch im DPBereich generell. Frequenz ist keine inhärente Eigenschaft von sprachlichen Strukturen. Sie hat außersprachliche Ursachen und Bedingungen. Definite Ausdrücke sind in der kindgerichteten Sprache hochfrequent, da mit Kindern bis zum Alter von ca. 3 Jahren fast ausschließlich über Ereignisse und Gegenstände geredet wird, die im Hier und Jetzt gegeben sind. Mehr lässt sich aber über erwerbsrelevante Frequenzverhältnisse bei D-Formen nicht sagen, so lange man nicht weiß, ob und in welcher Weise die Wahrnehmung von Inputfrequenzen durch das Kind selektiv ist. Ob also - und gerade, wenn es um den Erwerb von Bedeutungen geht - spezifischere Faktoren in die Frequenzanalyse einbezogen werden, wie etwa der Charakter der Konstituenten, in denen D-Formen auftreten, und/oder die Wortstellung und/oder der Kasusrahmen, von dem ja die Funktion der einzelnen Argumente abhängt. Soweit ich sehe, gibt es dazu keine Analysen. ${ }^{18}$ Wer sich mit dem Erwerb definiter Ausdrücke etwas auskennt, wird vermutlich von folgenden Zahlen überrascht sein: Im SimoneKorpus findet sich im ersten halben Jahr der Produktion von D-Formen durch Simone (Alter 1;9-2;2) in den Äußerungen ihres Vaters Max 2272x das, 1887 x die, 1328x der, 487x den und 131 x dem. Bei Simone sind es 994x das, 124x die, 189x der, 52x den und 4x dem. Der proportionale Anteil der 3 frequentesten D-Formen (das, die, der) ist damit bei Simone (SIM) deutlich anders als beim Vater (VAT), ihrem Hauptkommunikationspartner in den Sprachaufnahmen (Abb. 5). Auch wenn man das aufgrund seiner vielfach deiktischen bzw. sachverhaltsbezogenen Verwendung aus der Betrachtung ausklammert, zeigt sich der hier besonders interessante Unterschied in der Verwendungshäufigkeit von die und der (Abb. 6). ${ }^{19}$

18 In der Erwerbsforschung sind solche gewissermaßen multiplen Distributionsbedingungen sprachlicher Strukturen im Input im competition model (s. accessibility, reliability, cue strength; Bates \& MacWhinney 1987) und im natürlichkeitstheoretischen Modell (s. Uniformität, Transparenz; Dressler \& Karpf 1995) berücksichtigt worden. Frequenz ist im competition model ein Faktor unter anderen und im natürlichkeitstheoretischen Modell ein sekundärer (d. h. „abgeleiteter") Faktor.

19 Der Vergleich bezieht sich auf alle Verwendungen der D-Formen. Er schließt also auch pronominale und pluralische Verwendungen ein. Daraus ergibt sich m. E. keine Verzerrung der Erwerbsbedingungen. Es gibt keine a priori Beschränkung auf adnominale oder singularische Kontexte in der Inputerfassung durch das Kind, und auch nicht in seiner Sprachproduktion. In Modellen, die die Gebrauchs- und Inputfrequenzperspektive als Erklärungsbasis ansetzen, und annehmen, dass im Spracherwerb frequenzgeleitet zunächst unanalysierte Strukturen aus dem Input übernommen werden, sind die Verwendungskontexte nach meinem Verständnis ohnehin sekundär. 
Simone-Korpus: $1 ; 9-2 ; 2$

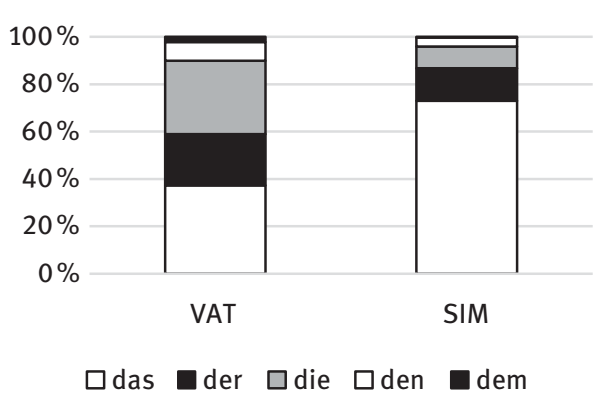

Abb. 5: Proportionale Häufigkeit der D-Formen.

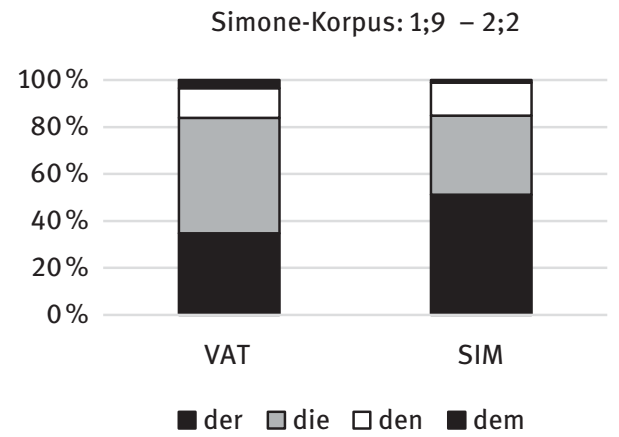

Abb. 6: Proportionale Häufigkeit der D-Formen ohne das.

Es ist leicht zu sehen, dass die Inputverhältnisse nicht unmittelbar den Erwerbsverlauf bedingen. Wäre das der Fall, sollte mindestens in Phase I die die-Form in allen Kontexten am häufigsten nicht-zielsprachlich verwendet werden. Argumente dafür, warum das nicht so ist, wurden in Abschnitt 3 dargelegt.

Eine nur scheinbare Möglichkeit einer konstruktionsgrammatischen Analyse des Erwerbs der D-Formen wäre die Postulation eines Konstruktionstyps „definites Nominal“, der verschiedene spezifischere Konstruktionen (DPs, DPronomen, definite PPs) umfasst. Eine solche Analyse würde sich jedoch nur noch in der Terminologie von einer strukturalistischen Analyse wie der oben präsentierten lexembasierten Analyse unterscheiden. 


\section{Fazit}

Soweit ich es beurteilen kann, gibt es gegenwärtig keinen konstruktionsgrammatischen Ansatz, der den Erwerb der D-Formen überzeugend erklären kann. Keine der im vorangegangenen Abschnitt diskutierten konstruktionsgrammatischen Annahmen zum Spracherwerb löst das zentrale Problem der Beschränkung des Hypothesenraumes. M.E. handelt es sich um ein grundsätzliches Problem, denn es ergibt sich aus der Annahme der Konstruktion als Basiseinheit der sprachlichen Strukturbildung. Für den Spracherwerb hieße dies, dass der Erwerb grammatischer Eigenschaften konstruktionsgebunden verläuft. In die Modellierung des Erwerbsprozesses müsste daher eingehen, welche Konstruktionen den Hypothesenraum für den Erwerb der D-Formen bilden und welche eventuell nicht dazugehören. Weiterhin müsste erfasst werden, wie sich die unterschiedlichen Eigenschaften der Konstruktionen im Hypothesenraum - insbesondere ihre verschiedenen konventionalisierten Bedeutungen - auf den Erwerbsprozess auswirken. Wie wird der Erwerbsprozess also davon beeinflusst, dass D-Formen in pronominalen, adnominalen, singularischen, pluralischen, nominativischen, akkusativischen usw. DP- und PP-Konstruktionen auftreten. Schließlich wäre zu klären, welche Bedeutungen/Funktionen den einzelnen D-Formen zukommen, so dass sie als sprachliche Zeichen im Sprachwissen verankert werden können. Die Definition der Konstruktion als konventionalisiertes Form-Bedeutungspaar hat vermutlich auch die Konsequenz, dass nur sehr begrenzt synkretistische Konstruktionen mit unterspezifizierten Bedeutungen angenommen werden können. Für Ausdrücke wie die warme Sonne müssen daher mindestens zwei Konstruktionsbedeutungen (also die_Xsg/Subjekt und die_Xsg/Objekt) erworben werden. Im Ergebnis ist ein sehr komplexes und statisches Ensemble von Konstruktionen $\mathrm{zu}$ erwerben. $\mathrm{Ob}$ dies durch den Mechanismus der Konstruktionsvererbung verhindert werden kann, ist fraglich. Spätestens mit der Zulassung von Einzelwörtern als Konstruktion übernimmt die Konstruktionsgrammatik strukturalistische Strukturprinzipien und gerät in Gefahr, ihre alternative Grammatikkonzeption aufzugeben.

Im Rahmen eines funktional-strukturalistischen Ansatzes lässt sich der Erwerbsprozess als schrittweise Spezifikation der lexikalisch-grammatischen Eigenschaften der D-Formen abbilden. Der Erwerb dieser Eigenschaften macht die D-Formen zu sprachlichen Zeichen. Sie spezifizieren den Bedeutungsanteil, den jede D-Form in komplexere Konstruktionen einbringen kann. Der Hypothesenraum für den Erwerb der D-Formen wird durch das Sample der in derselben syntaktischen Position auftretenden sprachlichen Einheiten bestimmt. Der zentrale Erwerbsprozess ist die Ermittlung der Funktionsoppositionen zwischen den Formen, deren syntaktische Distribution (partiell) identisch ist. Anhand 
der nicht-zielsprachlichen Verwendungen lässt sich zeigen, dass im Erwerbsprozess nicht zwischen D-Formen in pronominalen und adnominalen Konstruktionen unterschieden wird, sondern ein einheitlicher Verlauf vorliegt, in dem die paradigmatischen Relationen zwischen den einzelnen D-Formen hergestellt werden.

Es ist nicht überraschend, dass es bis heute wenige konstruktionsgrammatische Analysen morphologischer und speziell flexionsmorphologischer Strukturen gibt. Für die Konstruktionsgrammatik stellen paradigmatische Strukturen ein ernstes Problem dar, denn Mitglieder eines Paradigmas - oder, wenn man den Begriff Paradigma vermeiden will: einer funktionalen Domäne - sind durch spezifische Merkmale voneinander unterschieden. Streng konstruktionsgrammatisch muss das zur Differenzierung der Konstruktionsbedeutungen führen, denn paradigmatische Unterschiede sind Unterschiede in Form und Bedeutung von Ausdrücken. Werden die Formen eines Paradigmas aber nur im Rahmen von Konstruktionen erfasst, werden ihre paradigmatischen Relationen sekundär. Jede komplexere Konstruktion enthält so viel mehr (bzw. so transformierte) Bedeutungsanteile als die sie konstituierenden Einzelzeichen, dass deren Beitrag auf der Ebene der Konstruktionsbedeutung geradezu aus dem Blick fallen muss. Hier liegt m. E. ein Teil der Antwort auf die Frage, welche theoretischen Annahmen des Schema-Modells und des usage-based-Modells beim Zusammentreffen mit der Konstruktionsgrammatik aufgegeben wurden. Das Schema-Modell in der Version von Bybee (1985) beruhte auf der Annahme, dass ein separat von den sprachlichen Zeichen gespeicherter Regelapparat psychisch nicht real ist und schlug als alternatives Modell Netzwerkstrukturen zwischen den sprachlichen Zeichen bis hinunter zum Phonem vor. Angenommenen wurde ein (neuronales) Netzwerk, in dem gewissermaßen alles miteinander in formaler und funktionaler Relation steht. Wie stark die Relationen jeweils sind, hängt vom Sprachgebrauch ab, ist also usage-based. So stehen zum Beispiel alle Pluralformen miteinander in Relation so wie auch alle D-Formen. D. h. das Netzwerk enthält das Wissen über die gemeinsame (!) Zugehörigkeit von Einzelzeichen zu Funktionen oder von lexikalischen Wörtern zu semantischen Domänen. Mit der Etablierung der Konstruktion als sprachstrukturelle Basiseinheit wird die Relevanz der Einzelzeichen inklusive der Morpheme und ihrer paradigmatischen Relationen für die Bedeutungskonstitution negiert.

Das usage-based-Modell des Spracherwerbs stand der Konstruktionsgrammatik von Beginn an näher, indem es auf syntaktische Strukturen und das sogenannte holistische Lernen fokussierte. Hier wurden ursprünglich im Input frequent auftretende Konstruktionen als Ausgangspunkt des Lernprozesses verstanden. Der Lernprozess beinhaltete die Aufhebung ihrer spezifischen Bedeutungen durch Segmentierung, Generalisierung und Überführung in abstraktere 
Strukturmuster. Der Erwerb der D-Formen könnte danach so verstanden werden, dass die an Subjekt- bzw. Objekteigenschaften assoziierte Bedeutung der der- und den-Formen durch die (spätere) Erfassung der weiteren Verwendungsbereiche weiter generalisiert wird, so dass einerseits die synkretistische Verwendung von der und andererseits die Beschränkung von den auf Akkusativ Singular und Dativ Plural plausibel erfasst wird. In der Konstruktionsgrammatik sind Konstruktionen aber das Ziel, der Endpunkt des Erwerbsprozesses. Da sie gleichzeitig oberflächennah sind, sind Generalisierungsprozesse nicht mehr vollständig motiviert und vor allem ist die Beschränkung des Hypothesenraumes nicht als systematischer Abgrenzungsprozess und notwendiger Bestandteil von Generalisierung erfassbar. Wie vage die Bedingungen für Generalisierungen in dieser Situation werden, zeigt sich in der Formulierung von Generalisierungsbeschränkungen im konstruktionsgrammatisch orientierten usage-based-Modell (vgl. Tomasello 2008: 30, Behrens 2009: $433 \mathrm{ff}$.). ${ }^{20}$ Tomasello (2008: 31) räumt (trotz Goldberg 2006, DB) ein, „dass über die genaueren Einzelheiten solcher Beschränkungen des Generalisierungsprozesses sehr wenig bekannt ist“.

Mein zusammenfassendes Statement ist: Die mit der Etablierung der Konstruktionsgrammatik erfolgten theoretischen Entwicklungen erbringen zumindest für die Erfassung des Erwerbs paradigmatischer Relationen keinen Erkenntnisgewinn. Die Subsumierung paradigmatischer Relationen auf der Konstruktionsebene birgt die Gefahr der Negierung eines intern paradigmatisch (und syntagmatisch) strukturierten Sprachsystems. Es überrascht nicht, dass der Konstruktionsbegriff von Konstruktionsgrammatikern unterschiedlich gefasst und zunehmend kontrovers diskutiert wird (vgl. die Aufsatzsammlungen in Lasch \& Ziem 2011 und im Oxford Handbook of Construction Grammar herausgegeben von Hoffmann \& Trousdale 2013). Das Ziel einer Erfassung aller sprachlichen Strukturen auf einer einheitlichen Basis, in diesem Fall als Konstruktion, ist m. E. generell auf seine Angemessenheit hin $\mathrm{zu}$ hinterfragen. Vermutlich bestreiten aber auch heute wenige Forscher „dass analytische Fähigkeiten, die die Dekomposition eines komplexen Ausdrucks ermöglichen, und Kategorisierungen, die auf holistische Repräsentationen zurückzuführen sind, koexistieren“ (s. Eingangszitat). Die Dominanz der theoretischen Extreme hält dies jedoch im Verborgenen.

20 Generalisierungen liegt im usage-based Modell das sogenannte entrenchment zugrunde, das Einschleifen bzw. Verstärken von Mustern im Sprachgebrauch (Braine \& Brooks 1995; Tomasello 2009). Für eine kritische Auseinandersetzung mit der empirischen und der theoretischen Ausarbeitung des entrenchment-Konzepts s. Blumenthal-Dramé (2012). 


\section{Literatur}

Ágel, Vilmos \& Dagobert Höllein (i. d. B.): Satzbaupläne als Zeichen: die semantischen Rollen des Deutschen in Theorie und Praxis.

Bates, Elizabeth \& Brian MacWhinney (1987): Competition, variation, and language learning. In Brian MacWhinney (Hrsg.), Mechanisms of language acquisition, 157-193. Hillsdale, NJ: Lawrence Erlbaum.

Behrens, Heike (2009): Konstruktionen im Spracherwerb. Zeitschrift für Germanistische Linguistik 37, 427-444.

Bierwisch, Manfred (1967): Syntactic features in morphology: General problems of so-called pronominal inflection in German. To honour Roman Jakobson. Essays on the occasion of his seventieth birthday 11 October 1966. Vol. I, 239-270. The Hague, Paris: Mouton.

Bittner, Dagmar (1998): Entfaltung grammatischer Relationen im NP-Erwerb: Referenz. Folia Linguistica XXXI, 255-283.

Bittner, Dagmar (2002): Semantisches in der pronominalen Flexion des Deutschen. Zeitschrift für Sprachwissenschaft 21/2, 196-233.

Bittner, Dagmar (2004): The acquisition of polyfunctional forms - assignment of case and gender in German. Poster, Child Language Seminar, Bristol.

Bittner, Dagmar (2006): Case before gender in the acquisition of German. Folia Linguistica XL, 115-134.

Bittner, Dagmar (2007): Early functions of definite determiners and DPs in German first language acquisition. In Elisabeth Stark, Elisabeth Leiss \& Werner Abraham (Hrsg.), Nominal determination. Typology, context constraints and historical emergence, 215-240. Amsterdam, Philadelphia: Benjamins (Studies in Language Companion Series 89).

Blumenthal-Dramé, Alice (2012): Entrenchment in usage-based theories. What corpus-data do and do not reveal about the mind. Berlin: De Gruyter Mouton.

Braine, Martin S. \& Patricia J. Brooks (1995): Verb argument structure and the problem of avoiding an overgeneral grammar. In Michael Tomasello \& Willaim E. Merriman (Hrsg.), Beyond names for things: Young children's acquisition of verbs. Hillsdale, NJ: Erlbaum.

Bybee, Joan L. (1985): Morphology: a study of the relation between meaning and form. Amsterdam: Benjamins.

Bybee, Joan L. (2007): Frequency of use and the organization of language. Oxford: Oxford University Press.

Bybee, Joan L. (2013): Usage-based theory and exemplar representation. In Thomas Hoffman and Graeme Trousdale (Hrsg.), The Oxford handbook of construction grammar, 49-69. Oxford University Press.

Bybee, Joan, Revere Perkins \& William Pagliuca (1994): The evolution of grammar: Tense, aspect and modality in the languages of the world. Chicago: University of Chicago Press.

Clahsen, Harald (1984): Der Erwerb von Kasusmarkierungen in der deutschen Kindersprache. Linguistische Berichte 89, 1-31.

Coseriu, Eugenio (1975): Determinierung und Umfeld. In Eugenio Coseriu (Hrsg.), Sprachtheorie und Allgemeine Sprachwissenschaft. 5 Studien, 253-290. München: Fink.

Croft, William (2001): Radical construction grammar. Oxford: Oxford University Press.

Dressler, Wolfgang U. \& Annemarie Karpf (1995): The theoretical relevance of pre- and protomorphology in language acquisition. In Geert Booij \& Jaap van Marle (Hrsg.), Yearbook of Morphology 1994, 99-122. Springer: Dordrecht. 
Fischer, Kerstin \& Anatol Stefanowitsch (2006): Konstruktionsgrammatik: Ein Überblick. In Kerstin Fischer \& Anatol Stefanowitsch (Hrsg.), Konstruktionsgrammatik I. Von der Anwendung zur Theorie, 3-17. Tübingen: Stauffenburg.

Goldberg, Adele E. (1995): Constructions: A construction grammar approach to argument structure. Chicago: University of Chicago Press.

Goldberg, Adele E. (2006): Constructions at work: The nature of generalization in language. Oxford University Press on Demand.

Grießhaber, Wilhelm (i.d.B.): Muster und Frequenz: Nominalflexion nach Sprachkenntnissen und Sprachstatus.

Höhle, Barbara (2015): Crosslinguistic perspectives on segmentation and categorization in early language acquisition. In Edith Bavin \& Letitia Naigles (Hrsg.), The Cambridge handbook of child language. Second edition, 159-182. Cambridge: University Press.

Hoffmann, Thomas \& Graeme Trousdale (2013): The Oxford handbook of construction grammar. Oxford, New York: Oxford University Press.

Jakobson, Roman (1936/1971): Beitrag zur allgemeinen Kasuslehre. Gesamtbedeutungen der russischen Kasus. In Jindrich Toman \& Stephen Rudy (Hrsg.), Selected Writings II, 23-71. The Hague: Mouton.

Köpcke, Klaus-Michael (1993): Schemata bei der Pluralbildung im Deutschen. Versuch einer kognitiven Morphologie. Tübingen: Narr.

Koster, Jan (2017): Second thoughts about the Chomskyan revolution. In Martijn Wieling, Martin Kroon, Gertjan van Nord \& Gosse Bouma (Hrsg.), From semantics to dialectometry: Festschrift in honor of John Nerbonne (Tributes 32), 199-209. UK: College Publications.

Lasch, Alexander \& Alexander Ziem (Hrsg.) (2011): Konstruktionsgrammatik III. Aktuelle Fragen und Lösungsansätze. Tübingen: Stauffenburg.

Leiss, Elisabeth (1992): Die Verbalkategorien des Deutschen. Ein Beitrag zur Theorie der sprachlichen Kategorisierung. Berlin, New York: De Gruyter.

Mattes, Veronika (2018): Derivationsmorphologie und Wortarten im Erstspracherwerb des Deutschen. Unveröffentlichte Habilitationsschrift, Universität Graz.

Müller, Gereon (2003): Zwei Theorien der pronominalen Flexion im Deutschen. Deutsche Sprache 30, 328-363.

Müller, Natascha (1994): Gender and number agreement within DP. In Jürgen M. Meisel (Hrsg.), Bilingual first language acquisition. French and German grammatical development, 53-88. Amsterdam: Benjamins.

Pine, Julian M., Daniel Freudenthal, Gzregorz Krajewski \& Fernand Gobet (2013): Do young children have adult-like syntactic categories. Zipf's law and the case of the determiner. Cognition 127 (3), 345-360.

Radden, Günter (i. d. B.): Der verkannte $e$-Dativ im heutigen Deutsch: im Kreise meiner Lieben und am Rande des Abgrunds.

Suchodoletz, Waldemar von (2010): Daten zum Wortschatz von 20-25 Monate alten Kindern. https://www.kjp.med.uni-muenchen.de/download/Wortschatz_Text.pdf (04.12.2018).

Tomasello, Michael (1992): First Verbs: A case study of early grammatical development. Cambridge: Cambridge University Press.

Tomasello, Michael (2003): Constructing a language: A usage-based theory of language acquisition. Cambridge, MA: Harvard University Press.

Tomasello, Michael (2008): Konstruktionsgrammatik und früher Erstspracherwerb. In Anatol Stefanowitsch \& Kerstin Fischer (Hrsg.), Konstruktionsgrammatik II. Von der Konstruktion zur Grammatik, 19-37. Tübingen: Stauffenburg. 
Tomasello, Michael (2009): The usage-based theory of language acquisition. In Edith Bavin (Hrsg.), The Cambridge handbook of child language, 69-88. Cambridge: Cambridge University Press.

Tracy, Rosemarie (1986): The acquisition of case morphology in German. Linguistics 24, 47-78.

Wegener, Heide (1995): Das Genus im DaZ-Erwerb. Beobachtungen an Kindern aus Polen, Rußland und der Türkei. In Brigitte Handwerker (Hrsg.), Fremde Sprache Deutsch, 1-24. Tübingen: Narr.

Wegener, Heide (2000): German gender in children's second language acquisition. In Barbara Unterbeck, Matti Rissanen, Terttu Nevalainen \& Mirja Saari (Hrsg.), Gender in grammar and cognition, 511-544. Berlin: De Gruyter.

Weinert, Sabine \& Hannelore Grimm (2012): Sprachentwicklung. In Wolfgang Schneider \& Ulman Lindenberger (Hrsg.), Entwicklungspsychologie, 433-456. Weinheim: Beltz. 
